\title{
Music and Dementia: Individual Differences in Response to Personalized Playlists
}

\author{
Sandra Garrido ${ }^{\mathrm{a}, \mathrm{b}, \mathrm{d}, *}$, Catherine J. Stevens ${ }^{\mathrm{a}, \mathrm{b}}$, Esther Chang ${ }^{\mathrm{c}}$, Laura Dunne L $^{\mathrm{a}}$ and Janette Perz ${ }^{\mathrm{d}}$ \\ ${ }^{a}$ MARCS Institute for Brain, Behaviour and Development, Western Sydney University, Australia \\ ${ }^{\mathrm{b}}$ School of Social Sciences and Psychology, Western Sydney University, Australia \\ ${ }^{\mathrm{c}}$ School of Nursing and Midwifery, Western Sydney University, Australia \\ ${ }^{\mathrm{d}}$ Translational Health Research Institute, Western Sydney University, Australia
}

Handling Associate Editor: Amy Clements-Cortes

Accepted 11 May 2018

\begin{abstract}
Personalized music playlists are increasingly being used in health-care contexts to address the psychological and behavioral symptoms in people with dementia. However, there is little understanding of how people with different mental health histories and symptoms respond differently to music. A factorial experiment was conducted to investigate the influence of depression, anxiety, apathy, and cognitive decline on affective response to music. Ninety-nine people with dementia listened to three music playlists based on personal preferences. Activation of facial action units was measured, and behavioural responses continuously observed. Results demonstrated that people with high levels of depression and with symptoms of Alzheimer's type dementia demonstrated increased levels of sadness when listening to music. People with low depression but high levels of apathy demonstrated the highest behavioral evidence of pleasure during music listening, although behavioral evidence declined with severity of cognitive impairment. It is concluded that as well as accounting for personal preferences, music interventions for people with dementia need to take mental health history and symptoms into account.
\end{abstract}

Keywords: Dementia, depression, individual differences, music, playlists

\section{INTRODUCTION}

Music is frequently used as a non-pharmacological approach to managing behavioral and psychological symptoms of dementia (BPSD) [1]. Music interventions can include music therapy in which a registered music therapist facilitates programs of musical engagement that are either active (playing instruments, singing) or receptive (listening). The use of pre-recorded music without the involvement of a trained music therapist is also popular given the relative ease of access and cost-effectiveness of pre-recorded music. In particular, recent studies have examined the effectiveness of 'personalized playlists'

\footnotetext{
*Correspondence to: Dr. Sandra Garrido, The MARCS Institute for Brain, Behaviour \& Development, Western Sydney University, Locked Bag 1797, Penrith NSW 2751, Australia. Tel.: +61 9772 6585; E-mail: s.garrido@westernsydney.edu.au.
}

(PP) in which individual playlists are created for each patient based on their favorite music [2, 3].

PP hold some advantages over playing music to groups without consideration for individual tastes [4], and have generally positive effects on affective states [5]. However, not all people with dementia are favorably affected when listening to music that is personally significant. Garland and colleagues [3], for example, reported widely divergent responses, with dramatic reductions in agitation in some participants being "offset by neutral or negative outcomes for others" (p. 250). Other studies similarly report a worsening of mood or a neutral response in some participants [6], while others report no significant improvements at all [7].

One reason for this may be that merely selecting music that is a personal favorite of the individual does not ensure that the music is suitable for the therapeutic 
outcomes intended, nor does it take into account the mental health of the listener. For example, it has been found that different types of music can either diminish anxiety [8] or provoke anxiety in the listener [9].

Evidence further indicates that people with a history of mood disorders often respond differently to music to others and may be vulnerable to adverse responses. Studies in people without dementia suggest that mood disorders such as depression can complicate affective responses to music. Garrido and Schubert [10-12], for example, have shown that people with depression are particularly vulnerable to the triggering of dysphoric moods from sad music, but are more attracted to it than to other types of music. Thus, an individual with a history of depression may have 'favorite' music that is unlikely to produce positive mood outcomes. People with depression also find it more difficult to recover from dysphoria, rendering them vulnerable to long-term mood impacts. On the other hand, for people with dementia without a history of depression but who are currently withdrawn and apathetic, listening to preferred music can be a useful way to increase engagement with the world around them [13].

There is also little understanding of how different types of cognitive impairment influence affective response to music. For example, Alzheimer's disease usually involves extensive memory impairment and higher levels of depression than other forms of dementia [14]. This could leave people with Alzheimer's disease more susceptible to the activation of distressing memories. On the other hand, frontotemporal dementia is often associated with behavioral disturbances [14], and individuals with this type of dementia could be distracted or calmed by music. Furthermore, there is little information about how the degree of cognitive impairment influences response, with some studies suggesting that the effectiveness of music interventions tends to decrease with severity of impairment [13].

The aim of the current study was therefore to investigate how the psychological symptoms and mental health history of the patient influences their affective response to personalized playlists.

\section{Hypotheses}

H1: Sadness (indicated by facial expressions) increases from baseline when listening to music in people with high levels of depression and anxiety compared to people with low levels of depression and anxiety.
$\mathrm{H} 2$ : Pleasure (inferred from observational data) is significantly higher when listening to music for people with high levels of apathy but low depression.

H3: Pleasure is significantly lower when listening to music in people with severe cognitive decline compared to people with mild cognitive decline.

While no specific hypotheses were formulated, we also wanted to explore whether responses differed according to the type of dementia symptoms individuals presented with.

\section{METHODS}

\section{Study design}

A mixed factorial design was used to investigate the independent effect of four factors (independent variables): depression (high and low), anxiety (high and low), apathy (high and low), and cognitive decline (mild, moderate and severe), on mood (measured by facial expressions) and behavioral indicators of pleasure when listening to music.

\section{Participants}

We recruited 117 participants from six residential aged care facilities (nursing homes) in NSW, Australia. Of these, 113 met the eligibility criteria: a score of $<25$ on the Standardized Mini-Mental State Examination (SMMSE) [15], indicating the presence of mild to severe cognitive impairment [16], and no uncorrected hearing disorder that could prevent listening to music. Of these 113, one participant withdrew and 13 were unable to complete the study due to illness. A total of 99 people took part including 67 females and 32 males, aged 63 to 99 years (mean $[\mathrm{m}]=84$ years, standard deviation $[\mathrm{SD}]=8.1$ ) (Fig. 1).

Clinical diagnosis of dementia sub-type could only be obtained for 10 participants. Of those, three had been diagnosed with Alzheimer's dementia, three with vascular dementia, two with alcohol-induced dementia, one with Korsakoff's disease, and one with mild cognitive impairment. The rest of the participants had a non-specific diagnosis of dementia from a doctor or geriatrician. Scores on the SMMSE ranged from 0 to 25 with the average score being 7.7, indicating severe levels of cognitive impairment. Only 20 of the participants had any musical experience $(\mathrm{m}=42$ years, $\mathrm{SD}=27.7$ ). 


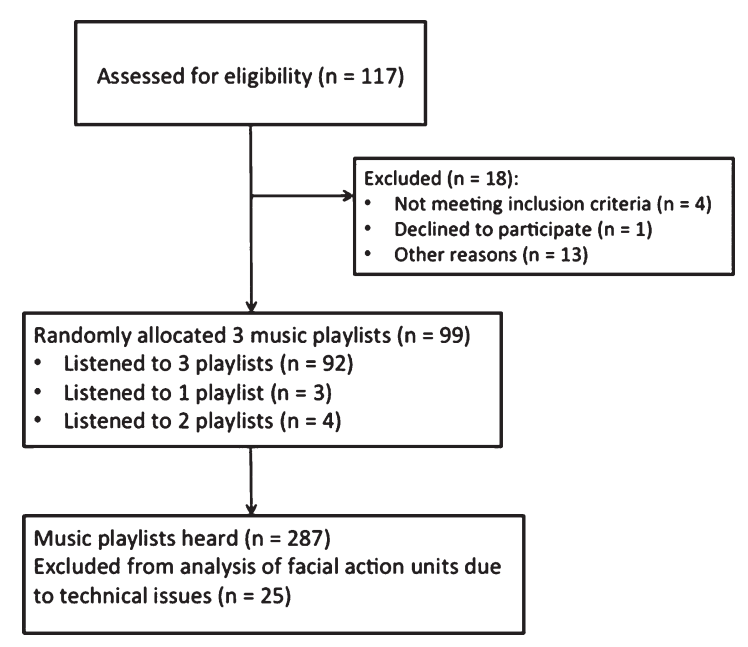

Fig. 1. Consort Flow chart of procedure.

\section{Procedures}

After obtaining ethics approval, facilities were randomly selected from a list and approached by phone. Participants from three facilities were recruited from this list, and from another three facilities by word of mouth. After referral to the study by staff and obtaining consent from individuals and/or legal guardians, a family member or caregiver completed a prescreening questionnaire. Where possible participants themselves completed a questionnaire about musical preferences and a test of cognitive functioning. Caregivers or family members provided information about music preferences where participants were too severely impaired to provide this information themselves. From this information musical preferences were assessed and exclusion criteria checked.

One to two weeks after completion of the prescreener, eligible participants took part in a listening session lasting approximately 30 minutes at their facility. Participants were randomly allocated 3 music playlists of 8-9 minutes each with 2-3 minutes between conditions and a 2 -minute baseline period ( 7 participants listened to 1-2 playlists only). Sessions were usually conducted in a private location, although 15 participants were accompanied by a family member.

\section{MEASURES AND MATERIALS}

\section{Pre-screen measures}

The pre-screener consisted of questions from Gerdner's Assessment of Personal Music Preference (the patient or family versions) [17], an assessment of cognitive functioning (SMMSE) [15], the 9-item Patient Health Questionnaire [PHQ-9; 18] for assessing depression, the 7-item Generalized Anxiety Disorder scale (GAD-7) [19] for assessing anxiety, and the Apathy Evaluation Scale [20] for measuring general apathy. Family members or caregivers were asked to complete the three latter scales on the basis of the individual's history and current behavior.

The SMMSE is widely used as a measure of cognitive impairment [21]. It measures several domains of cognitive functioning including time and place orientation; short and long-term memory; constructional ability; language and ability to understand and follow instructions. Intraclass correlations have been reported as 0.90 [22], and validity has been reported as high when assessed against several alternative measures as well as geriatrician screenings [23, 24]. The SMMSE also holds the advantage that it is relatively short to administer, taking only approximately 10-12 minutes on average [21].

The PHQ-9 incorporates DSM criteria for assessing depression into a 9-item scale. It has been shown to have high internal consistency and test-retest reliability [25] and has been validated in older adults [26]. Studies have also found that proxy reports on this scale are often more reliable that self-reports, particularly when reporting observable behavioral symptoms of depression [27]. In the current sample reliability score was 0.84 (Chronbach's alpha). Similarly, the GAD-7 has demonstrated good reliability and validity as a screener for generalized anxiety disorder [28], as well as a measure of general anxiety symptoms in diverse populations [29] including in older adults [30]. The current sample returned a reliability score of 0.91 (Chronbach's alpha). The Apathy Evaluation Scale was specifically developed for use with patients with dementia and is available in three versions: for clinician, informant and self-rated [31]. The scale has been reported to have satisfactory levels of internal consistency, and test-retest and interrater reliability [31]. In the current study the reliability score was 0.92 (Chronbach's alpha).

\section{Musical stimuli}

A database of music from the 1930s-1970s was created with songs categorized according to tempo (speed), mode (key) and genre (e.g., classical, popular, country, etc.). Three individualized playlists were created for each participant based on their reported genre, song and artist preferences. For example, if an 
individual reported that they liked Frank Sinatra, and was assigned to the fast tempo, major mode category, an effort was made to find a Frank Sinatra song that fit those experimental criteria. Each playlist contained 2-4 songs matched according to tempo, and mode. This enabled us to avoid the possible confounding influence of genre. For example, country music may tend to be relatively slow. Therefore, if an individual reported a preference for country music an effort was made to ensure that they listened to country music in both a slow and a fast tempo in different playlists in order to ensure that affective responses did not occur primarily because of genre choice. 'Sad' music (i.e., slow music in minor keys and/or with negative lyrics) was excluded from the third playlist heard by individuals to avoid the possibility of participants ending the experiment in a negative mood.

Music was played on an Apple iPad 2 through a set of Sennheiser HDR 160 wireless headphones. Four participants preferred to listen without headphones and therefore heard the music directly from the iPad speakers.

\section{Experimental measures}

Facial expressions are a frequently used measure of affective response [32]. Whereas other biometric measures such as skin conductance or heart rate are useful indicators of arousal, activation of facial muscles have the advantage of being able to demonstrate the valence as well as intensity of affective responses [33]. Facial electromyography is one way of measuring affective responses through the movement of certain facial muscles [34]. However, since the use of electrodes can cause some discomfort or distress in people with dementia [35], automated systems for coding facial expressions from video footage are useful with this population [36].

In the current study, facial expressions were filmed continuously by a Logitech c 270 webcam positioned approximately one meter in front of the face of participants in good lighting conditions and connected via USB port to an Acer Inspire s7-392 laptop. A Sony Handycam HDR-PJ200 recorded the participant's whole body while listening to the music. Researchers rated behavioral responses from this video data that indicated the strength of memories and responses to the music as described in the subsequent section [37]. The musical stimuli and video data were synchronized using LabChart 8 . Between playlists participants who could do so $(n=82$, $82.8 \%$ ) rated familiarity of the music and indicated enjoyment on an adaptation of the Wong-Baker FACES scale [38].

\section{Analysis}

Facial expressions were analyzed using Noldus FaceReader 6 with the Action Unit Module. FaceReader uses a model-based method to analyze the varying location of 500 key points in the face and generates numerical output about the activation of 20 Action Units (AU) of the Facial Action Coding System [39]. It also generates composite scores of primary emotions such as Sadness and Happiness based on the activation of a combination of AUs. Studies have demonstrated that such automated coding can be more reliable than human coding [40, 41]. Individual data files were first calibrated using still shots of neutral facial expressions for each participant in order to account for personal variation in facial expression. Average scores over 30 -second baseline periods and 7-minute test blocks were obtained for AUs and composite emotions scores.

Whole body video data was coded independently by two coders using the Observed Emotion Rating Scale OERC, [42]. Interrater reliability in the current study was found to be Kappa $=0.83(p<0.001)$. The OERC has been used in many studies involving people with dementia, including studies about communication between spouses [43], the use of telepresence robots [44], and the potential of rhythmic sensory stimulation to increase alertness [45]. In the current study, time points in the data at which behaviors (such as laughing, singing, restlessness, tapping feet, or swaying) began and ended were noted. These behaviors were then categorized according to the instructions provided by the authors of the OERC as evincing Pleasure, Anger, Anxiety/Fear, Sadness, and general Alertness, and a total time score for each behavior was generated for each listening block.

Scale scores from the pre-screener data were calculated according to scale protocols. Scores on SMMSE items were also used to determine the possible type of dementia participants may have. Vertesi and colleagues [21] outlined particular items of the SMMSE that can indicate the differential deteriorations that occur in Alzheimer's disease, vascular dementia, and dementia with Lewy bodies. For example, people with Alzheimer's disease tend to perform more poorly on items relating to short-term memory, while people with dementia with Lewy bodies may perform more poorly on visuospatial items. For participants 
in our study for whom we were not able to obtain a specific diagnosis, patterns of answers were considered in order to determine a possible dementia sub-type and participants for whom patterns were clear were allocated to the Alzheimer's, vascular or dementia with Lewy bodies category. Combined with those participants who had formal diagnoses this enabled us to infer a diagnosis for a total of 48 participants. Subsequent data analyses were conducted using SPSS 22.0

\section{RESULTS}

Participants reported that the music they heard was relatively familiar $(\mathrm{m}=3.5, \mathrm{SD}=1.4$ range $1-5)$, and enjoyable $(\mathrm{m}=4.0, \mathrm{SD}=1.2$ range $1-5)$. Researcher observation ratings of the strength of memories evinced by the music [37] showed responses ranging from no sign of recognition but some sign of interest, to showing a weak sense of familiarity via verbal responses, humming or facial expressions. No participants verbalized any specific memories associated with the music they were played.

\section{Influence of depression, anxiety, and apathy}

To investigate the influence of depression and anxiety on facial expressions a repeated measures ANOVA was conducted with Sadness (measured by FaceReader) as the dependent variable and Time as the within-subjects factor (baseline, test block). Scores on depression and anxiety were split at the median to form Depression group (high and low) and Anxiety group (high and low) and entered as the between-subjects variable. Familiarity was added as a covariate. The median score of depression was 11 -considered to be an acceptable cut-off score for the PHQ-9 [46], with a median of 14 for anxiety-generally considered to be the cutoff between moderate and severe anxiety for the GAD-7 [28]. A significant main effect of Time was found on Sadness $F(1,231)=6.3, p=.013$, Wilks' lamda $=0.97, \eta \mathrm{p}^{2}$ (partial eta squared $)=0.03$. A significant three-way interaction was found between Time, Depression group and Anxiety group $F(1,213)=4.6, p=0.033$, Wilks' lamda $=0.98$, $\eta \mathrm{p}^{2}=0.02$. Post-hoc paired-samples $t$-tests revealed that the significant effect came from the group with low Anxiety and high Depression where facial expressions evincing sadness increased from baseline $(m=0.04, S D=0.06)$ to test block $(m=0.10$, $\mathrm{SD}=0.17)$ when listening to music $t(32)=-2.5$, $p=0.018$. No other group had significant changes over time (Figs. 2 and 3). There was no significant interactive effect for Familiarity $F(1,213)=0.01$, $p=0.93$, Wilks' lamda $=1.0, \eta \mathrm{p}^{2}<0.001$. Thus, Sadness increased significantly for people with high levels of depression. However, contrary to expectation, people with low levels of anxiety were more likely to experience increased Sadness when listening to music if they also experienced high depression.

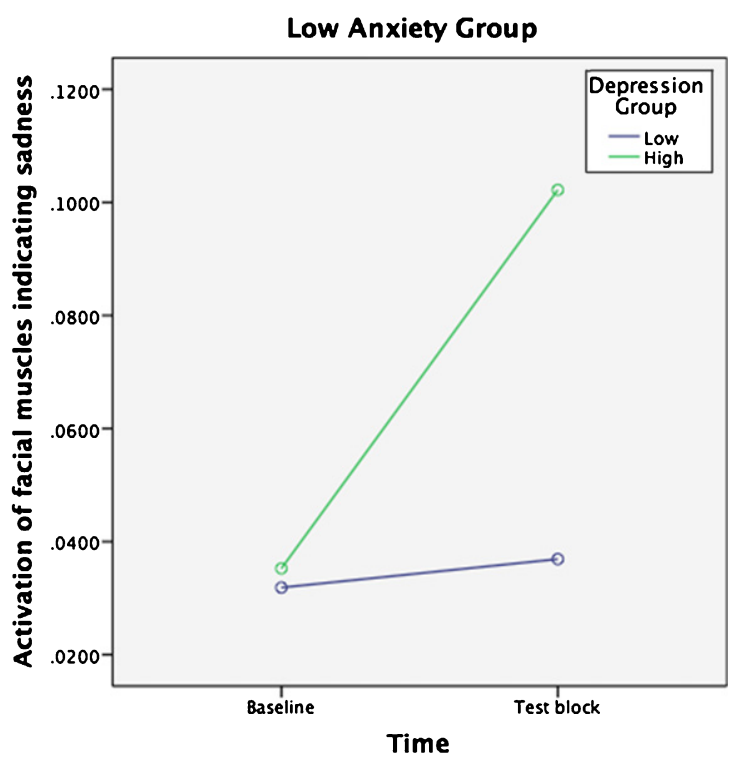

Fig. 2. Sadness scores over Time for low Anxiety group.

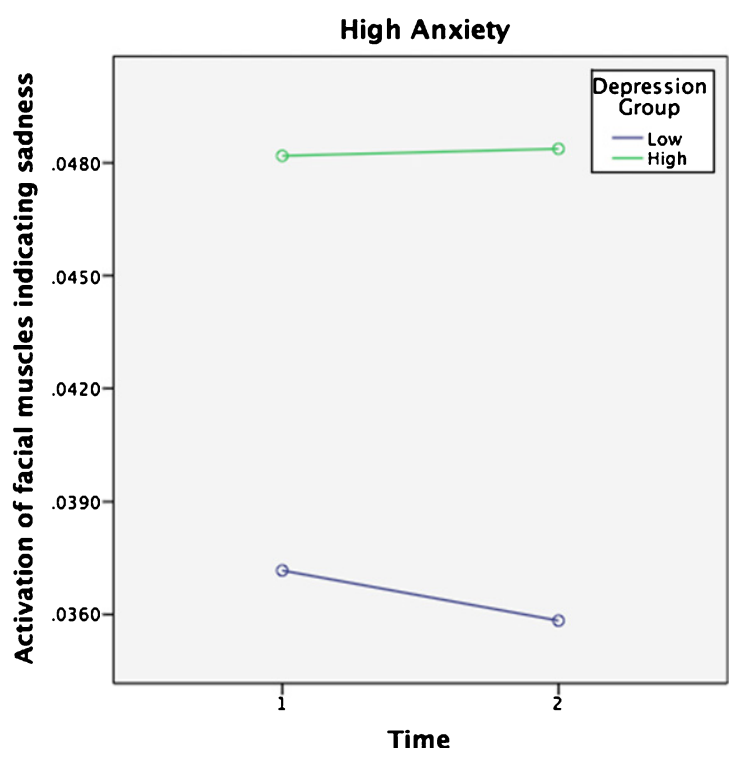

Fig. 3. Sadness scores over Time for high Anxiety group. 


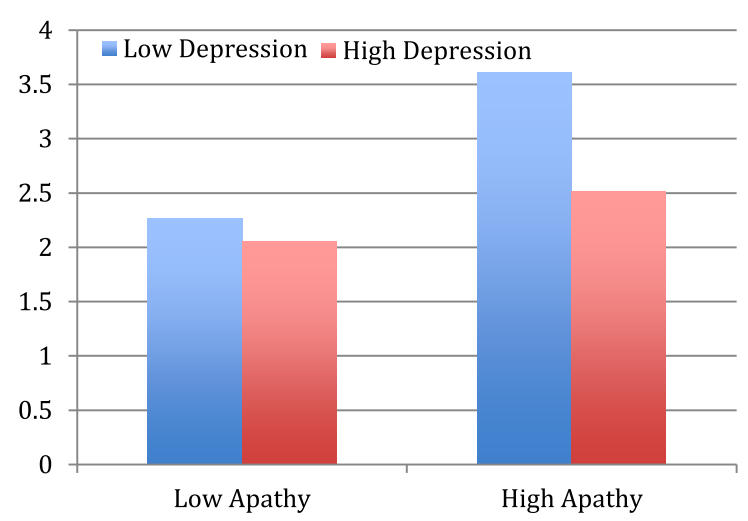

Fig. 4. Behavioral indications of Pleasure comparisons between Apathy and Depression Groups.

To investigate the effect of depression and apathy on pleasure when listening to music, a univariate analysis of variance was conducted with behavioral observation ratings of Pleasure as the dependent variable, and Apathy Group (low and high) and Depression Group (high and low) as independent variables. A significant main effect of Apathy Group $F(1,199)=19.2, p<0.001, \eta \mathrm{p}^{2}=0.1$ and Depression group $F(1,199)=10.1, p=0.002$, $\eta \mathrm{p}^{2}=0.1$ was found. A significant interaction was also found between Apathy and Depression Groups $F(1,199)=4.8, p=0.031, \eta \mathrm{p}^{2}=0.02$, with post -hoc analyses demonstrating that people with both high apathy and low depression demonstrated the highest behavioral evidence of pleasure when listening to music (Fig. 4). The second hypothesis was thus supported.

\section{Severity of cognitive impairment and type of dementia}

To test the influence of level of cognitive decline on pleasure when listening to music we conducted a univariate analysis of variance with behavioral observation ratings of Pleasure as the dependent variable, and Severity of Dementia Group (Mild, Moderate and Severe) as the independent variable. A significant main effect of Severity of Dementia Group was found $F(1,207)=6.1, p=0.003, \eta p^{2}=0.1$. Post-hoc Scheffe analyses showed that the significant differences $(p=0.011)$ existed between Mild $(\mathrm{m}=3.5$, $\mathrm{SE}=0.4)$ and Severe $(\mathrm{m}=2.3, \mathrm{SE}=0.1)$ dementia groups. These results indicate that people with severe cognitive impairment demonstrated lower levels of Pleasure in music listening, in support of the third hypothesis.
In order to explore the effect of type of dementia on affective response to music, a one-way ANCOVA was conducted with FaceReader Sadness scores at the test block as the dependent variable, Dementia Type (Alzheimer's, Lewy Bodies, or Vascular) as the independent variable, and FaceReader Sadness scores at baseline as the covariate. A significant main effect of Dementia Type was found $F$ (2, 110) $=4.4, p=0.015, \eta p^{2}=0.1$. Post - hoc analyses showed that the significant differences (Scheffe $p=0.015$ ) lay between people with symptoms of Alzheimer's and vascular dementias. With Sadness scores at Baseline controlled for, a comparison of estimated marginal means showed that people with symptoms of Alzheimer's $(\mathrm{m}=0.08, \mathrm{SE}=0.01)$ demonstrated higher levels of Sadness during music listening than people with symptoms of Vascular dementia $(m=0.02, \mathrm{SE}=0.02)$.

\section{DISCUSSION}

We had hypothesized that people with high levels of depression and anxiety were more likely to experience adverse affective responses to music, while people with low depression who were withdrawn and apathetic would experience more positive responses. In addition, we had predicted that people with more severe levels of cognitive impairment would demonstrate reduced responsiveness to music. These hypotheses were for the most part supported.

Results revealed that people with high levels of depression had increased facial expressions indicating sadness while listening to music compared to baseline. Depression is associated with a negative attentional bias, meaning that people with a history of depression are more likely to interpret stimuli in negative ways [47] and to recall negative memories [48]. Evidence suggests that this also occurs when listening to music, with studies demonstrating that music often triggers negative memories or thoughts in people with tendencies to depression [49]. The current study indicated that this may also occur in people with dementia, since those with a history or current symptoms of depression seemed to experience increased sadness when listening to music compared to people with low levels of depression.

However, this was particularly so in people who also had low anxiety. While we had anticipated that people with high anxiety would experience negative emotions in response to music, this was not borne out by the data. While this finding is con- 
trary to some studies relating to populations without dementia which have found that music can sometimes increase anxiety in people with anxiety disorders [50], one of the most consistent findings in relation to the use of PP with people with dementia is the reduction of agitation [5]. Thus people with dementia who have a history or symptoms of depression may be particularly susceptible to the triggering of negative affect when listening to music. However, in the case of anxiety or agitation, music may provide more of a distractive benefit, tending to re-direct attention away from distressing thoughts or feelings.

Conversely in the current study, people with low scores in depression who were apathetic appeared to derive the most enjoyment from listening to music. Thus, while music may trigger negative affect in people with tendencies to depression, music appears to be a useful tool for helping people to re-engage with the world around them for those without the same vulnerability to negative responses. It may be that music is particularly enjoyable for people who are apathetic due to the increased mental stimulation music can provide. In addition, where music is relatively familiar and of personal significance to the individual it may help them to reconnect with their own history, providing a renewed sense of self in a clinical environment that may often be devoid of personal meaning [51].

Nevertheless, the results indicated that as cognitive impairment becomes more severe, pleasurable responses to music decrease. Whether or not this is reflective of decreased responsiveness to stimuli in general or of actually diminishing pleasure cannot be answered based on the results of the current study. In fact, one of the difficulties inherent in studies of this kind in people with dementia is the challenge of measuring the subjective experience of individuals who have a reduced capacity to communicate.

Another interesting finding in the current study was that people with different types of cognitive decline responded to music in different ways, with people likely to have Alzheimer's dementia showing a greater likelihood of experiencing negative affect in comparison to people with symptoms of vascular dementia. To our knowledge, this is the first study that has examined the varying response of different dementia sub-types to music. It is not surprising that music would affect people differently depending on their diagnosis, given the different behavioral patterns and brain regions effected in the dementia sub-types $[52,53]$. However, the current study was limited by the availability of clear diagnoses for all participants, another challenge associated with research in this field given the frequently non-specific diagnoses that many patients receive. Future research with participants who have undertaken neuropsychological tests or other robust diagnostic methods will be able to investigate this question more closely. Further limitations that are also common to studies in people with dementia was the use of proxy reports for measures of depression, anxiety, and apathy.

\section{Conclusion}

The current study demonstrates that the mere selection of 'favorite' or personally significant music may not be enough to ensure positive outcomes from music interventions. These results have implications for the use of music by care workers or music therapists in aged care settings, suggesting that it is important to select music with care and to have appropriate support systems in place when using music with vulnerable people with a history or current symptoms of depression.

Future interventions will need to take into account the psychological history and symptoms of the individual, considering in more detail how vulnerabilities to negative affect may be detrimental in people with tendencies to depression who may be slower to recover from negative emotions. Care may need to be taken to ascertain the history that individuals have with particular pieces of music and to select music that is most likely to connect individuals with happy memories and to evoke positive emotions. Future studies could also investigate individual differences in response to music from a neurological point of view, focusing on the potential for neurorehabilitative effect.

\section{ACKNOWLEDGMENTS}

This research was supported by a Fellowship to the first author from the National Health and Medical Research Council of Australia.

Authors' disclosures available online (https:// www.j-alz.com/manuscript-disclosures/18-0084r2).

\section{REFERENCES}

[1] Mitchell G, Agnelli J (2015) Non-pharmacological approaches to alleviate distress in dementia care. Nurs Standard 30, 38-44.

[2] Sung HC, Chang AM, Abbey J (2006) The effects of preferred music on agitation of older people with dementia in Taiwan. Int J Geriatr Psychiatry 21, 999-1000. 
[3] Garland K, Beer E, Eppingstall B, O'Connor DW (2007) A comparison of two treatments of agitated behaviour in nursing home residents with dementia: Simulated family presence and preferred music. Am J Ger Psychiat 15, 514521.

[4] Nair BK, Heim C, Krishnan C, D'Este C, Marley J, Attia J (2011) The effect of Baroque music on behavioural disturbances in patients with dementia. Australas J Ageing 30, 11-15.

[5] Garrido S, Dunne L, Chang E, Perz J, Stevens C, Haertsch M (2017) The use of music playlists for people with dementia: A critical synthesis. J Alzheimers Dis 60, 1129-1142.

[6] Martin PK, Schroeder RW, Smith JM, Jones B (2016) The Roth project - Music and Memory: Surveying the observed benefit of personalized music in individuals with diagnosed or suspected dementia. Alzheimers Dement 12, P988.

[7] Kwak J, Brondino MJ, O'Connell Valuch K, Maeda H (2016) Evaluation of the Music and Memory program among nursing home residents with dementia: Final report to the Wisconsin Department of Health Services, University of Wisconsin-Milwaukee.

[8] Thompson M, Moe K, Lewis P (2014) The effects of music on diminishing anxiety among preoperative patients. J Radiol Nurs 33, 199-202.

[9] Harada T, Kurai R, Ito S, Nitta Y, Aoi S, Ikeda H, Iida T, Miyazaki H, Umei N, Chikamura C, Ishizaki F, Nitta $\mathrm{K}$ (2017) Effect of joyful and anxiety-provoking music on autonomic nervous system function. Int Med J 24, 211-213.

[10] Garrido S, Schubert E (2013) Adaptive and maladaptive attraction to negative emotion in music. Musicae Scientiae 17, 145-164.

[11] Garrido S, Schubert E (2015) Moody melodies: Do they cheer us up? A study of the effect of sad music on mood. Psychol Music 43, 244-261.

[12] Garrido S, Schubert E (2015) Music and people with tendencies to depression. Music Percept 32, 313-321.

[13] Holmes C, Knights A, Dean C, Hodkinson S, Hopkins V (2006) Keep music live: Music and the alleviation of apathy in dementia subjects. Int Psychogeriatr 18, 623-630.

[14] Cerejeira J, Lagarto L, Mukaetova-Ladinska EB (2012) Behavioral and psychological symptoms of dementia. Front Neurol 3, 73 .

[15] Molloy DW, Alemayehu E, Roberts RO (1991) Reliability of a standardized Mini-Mental State Examination compared with the traditional Mini-Mental state Examination. Am J Psychiatry 14, 102-105.

[16] Perneczky R, Wagenpfeil S, Komossa K, Grimmer T, Diehl J, Jurz A (2006) Mapping scores onto stages: Mini-mental state examination and clinical dementia rating. Am J Geriatr Psychiatry 14, 139-144.

[17] Gerdner LA (2000) Evidence-based protocol: Individualized music intervention, University of Iowa.

[18] Spitzer RL, Kroenke K, Williams JB (1999) Patient Health Questionnaire Study Group. Validity and utility of a selfreport version of PRIME-MD: The PHQ Primary Care Study. JAMA 282, 1737-1744

[19] Kroenke K, Lowe B, Spitzer RL, Williams JB (2006) A brief measure for assessing Generalized Anxiety Disorder: The GAD-7. Arch Intern Med 166, 1092-1097.

[20] Marin RS (1996) Apathy: Concept syndrome, neural mechanisms and treatment. Semin Clin Neuropsychiatry 1, 304-314.

[21] Vertesi A, Lever JA, Molloy DW, Sanderson B, Tuttle I, Pokoradi L, Principi E (2001) Standardized Mini-Mental
State Examination: Use and interpretation. Can Fam Physician 47, 2018-2023.

[22] Bedard M, Molloy DW, Standish T, Guyatt GH, D’Souza J, Mondadori C (1995) Clinical trials in cognitively impaired older adults: Home versus clinic assessments. J Am Geriatr Socy 43, 1127-1130.

[23] Molloy DW, McIlroy WE, Guyatt GH, Lever JA. (1991) Validity and reliability of the Dysfunctional Behavior Rating Instrument. Acta Psychiatr Scand $\mathbf{8 4}$.

[24] Molloy DW, Silberfeld M, Darzins P, Guyatt GH, Singer PA, Rush B (1996) Measuring capacity to complete advanced directives. J Am Geriatr Soc 44, 660-664.

[25] Fann JR, Bombardier CH, Dikmen S, Esselman P, Warms CA, Pelzer E, Rau H, Temking N (2005) Validity of the Patient Health Questionnaire-9 in assessing depression following traumatic brain injury. J Head Trauma Rehabilitation 6, 501-511.

[26] Phelan E, Williams B, Meeker K, Bonn K, Frederick J, LoGerfo J, Snowden M (2010) A study of the diagnostic accuracy of the PHQ-9 in primary care elderly. BMC Fam Pract 11, 63.

[27] Rooney AG, McNamara S, Mackinnon M, Fraser M, Rampling R, Carson A, Grant R (2013) Screening for major depressive disorder in adults with glioma using the PHQ-9: A comparison on patient versus proxy reports. J Neurooncol 113, 49-55.

[28] Spitzer RL, Kroenke K, Janet BW, Williams DSW, Lowe B (2006) A brief measure for assessing Generalized Anxiety Disorder: The GAD-7. Arch Intern Med 166, 1092-1097.

[29] Beard C, Björgvinsson T (2014) Beyond generalized anxiety disorder: Psychometric properties of the GAD-7 in a heterogeneous psychiatric sample. J Anxiety Disord 28, 547-552.

[30] Wild B, Eckl A, Herzog W, Niehoff D, Lechner S, Maatouk I, Schellberg D, Brenner H, Mueller H, Lowe B (2014) Assessing Generalized Anxiety Disorder in elderly people using the GAD-7 and GAD-2 scales: Results of a validation study. Am J Geriatr Psychiatry 22, 1029-1038.

[31] Marin RS, Biederzycki RC, Firinciogullari S (1991) Reliability and validity of the Apathy Evaluation Scale. Psychiatry Res 38, 143-162.

[32] Lewinski P, Fransen ML, Tan ESH (2014) Predicting advertising effectiveness by facial expressions in response to amusing persuasive stimuli. J Neurosci Psychol Econ 7, $1-14$.

[33] Cacioppo JT, Petty RE, Losch ME, Kim HS (1986) Electromyographic activity over facial muscle regions can differentiate the valence and intensity of affective reactions. J Pers Soc Psychol 50, 260-268.

[34] Manssuer LR, Pawling R, Hayes AE, Tipper SP (2016) The role of emotion in learning trustworthiness from eye-gaze: Evidence from facial electromyography. Cogn Neurosci 7, 82-102.

[35] Garrido S, Steiner GZ, Russo N (2018) People with dementia: The challenges for data collection with a vulnerable population. SAGE Research Methods Cases Part 2. doi: $10.4135 / 9781526439024$

[36] Girard JM, Cohn JF, Laszlo JA, Sayette M, De la Torre F (2015) Spontaneous facial expression in unscripted social interactions can be measured automatically. Behav Res Methods 47, 1136-1147.

[37] Samson S, Dellacherie D, Platel H (2009) Emotional power of music in patients with memory disorders: Clinical implications of cognitive neuroscience. Ann N Y Acad Sci 1169, 245-255. 
[38] Wong-Baker FACES Foundation (2016) Wong-Baker FACES Pain Rating Scale. http://www.WongBakerFACES. org

[39] Ekman P, Friesen WV (1986) A new pan-cultural facial expression of emotion. Motiv Emot 10, 159-168.

[40] Lewinski P (2015) Automated facial coding software outperforms people in recognizing neutral faces as neutral from standardized datasets. Front Psychol 6, 1386.

[41] Lewinski P, den Uyl TM, Butler C (2014) Automated facial coding: Validation of basic emotions and FACS AUs in FaceReader. J Neurosci Psychol Econ 7, 227-236.

[42] Lawton MP, Van Haitsma K, Klapper JA (1999) Observed affect and quality of life in dementia: Further affirmations and problems. J Ment Health Aging 5, 69-81.

[43] Williams CL, Newman D, Hammer LM (2017) Preliminary psychometric properties of the verbal and nonverbal interaction scale: An observational measure for communication in persons with dementia. Issues Ment Health Nurs 38, 381-390.

[44] Moyle W, Jones C, Cooke ML, O’Dwyer S, Sung B, Drummond S (2014) Connecting the person with dementia and family: A feasibility study of a telepresence robot. $B M C$ Geriatr 14, 1-11.

[45] Clements-Cortes A, Bartel L, Ahonen H, Freedman M (2017) The potential of rhythmic sensory stimulation treatments for persons with Alzheimer's disease. Music Med $\mathbf{9}$, 167-173.

[46] Manea L, Gilbody S, McMillan D (2012) Optimal cut-off score for diagnosing depression with the Patient Health Questionnaire (PHQ-9): A meta-analysis. Can Med Assoc J 184, E191-E196.
[47] Platt B, Murphy SE, Lau JYF (2015) The association between negative attentional biases and symptoms of depression in a community sample of adolescents. PeerJ 3, e1372.

[48] Dalgliesh T, Werner-Seidler A (2014) Disruptions in autobiographical memory processing in depression and the emergence of memory therapeutics. Trends Cogn Sci 18, 596-604.

[49] Garrido S, Bangert D, Schubert E (2016) Musical prescriptions for mood improvements: A mixed methods study. Arts Psychother 51, 46-53.

[50] Mennin DS, Heimberg RG, Turk CL, Fresco DM (2005) Preliminary evidence for an emotion dysregulation model of generalized anxiety disorder. Behav Res Ther 43, 12811310.

[51] Yasuda K, Kuwabara K, Kuwahara N, Abe S, Tetsutani N (2009) Effectiveness of personalised reminiscence photo videos for individuals with dementia. Neuropsychol Rehabil 19, 603-619.

[52] Du A-T, Schuff N, Kramer JH, Rosen HJ, Gorno-Tempini ML, Rankin K, Miller BL, Weiner MW (2007) Different regional patterns of cortical thinning in Alzheimer's disease and frontotemporal dementia. Brain 4, 1159-1166.

[53] Srinivasan S, Nagaraja AV, Ratnavalli E (2005) Neuropsychiatric symptoms in dementia - frequency, relationship to dementia severity and comparison in Alzheimer's disease, vascular dementia and frontotemporal dementia. J Neurol Sci 236, 43-48. 